\title{
A Novel Frequency Offset Estimation Algorithm Using Differential Combining for OFDM-Based WLAN Systems ${ }^{\star}$
}

\author{
Sangho Ahn ${ }^{1}$, Sanghun Kim ${ }^{1}$, Hyoung-Kee $\mathrm{Choi}^{1}$, Sun Yong $\mathrm{Kim}^{2}$, \\ and Seokho Yoon $^{1}$ \\ ${ }^{1}$ School of Information and Communication Engineering, Sungkyunkwan University, \\ 300 Chunchun-dong, Jangan-gu, Suwon, Kyunggi-do, 440-746, Korea \\ \{ash9252, hkchoi, ksh7150, syoon\}@ece.skku.ac.kr \\ 2 Department of Electronics Engineering, Konkuk University, \\ 1 Hwayang-dong, Gwangjin-gu, Seoul 143-701, Korea \\ kimsy@konkuk.ac.kr
}

\begin{abstract}
The timing offset is one of the main error sources in estimating the frequency offset in orthogonal frequency division multiplexing (OFDM)-based wireless local area network (WLAN) systems. Although some works have been done to mitigate the effect of the timing offset on the frequency offset estimation, most of the investigations require the knowledge of the timing offset range, which is not generally available in practical systems. In this paper, we propose a new frequency offset estimation algorithm using differential combining between two successive correlation samples, which does not require the knowledge of the timing offset range, and thus, is robust to the timing offset variation. The simulation results show that the proposed algorithm is not only robust to the timing offset variation, but also generally performs better than the conventional algorithm, in the case of the timing offset range being not known exactly.
\end{abstract}

Keywords: OFDM, WLAN, frequency offset estimation.

\section{Introduction}

Future wireless and mobile systems are envisioned to provide reliable and highspeed multimedia services to users. Among the wireless and mobile technologies proposed so far, OFDM technology has been attracting considerable research interest for wireless and mobile applications [1] and selected as a modulation scheme in wireless local area network (WLAN) standards, such as institute of electrical and electronics engineers (IEEE) 802.11a, high performance local area network type 2 (HiperLAN/2), and mobile multimedia access communication

\footnotetext{
* This research was supported by grant No. R01-2004-000-10690-0 from the Basic Research Program of the Korea Science \& Engineering Foundation. Dr. Yoon is the corresponding author.
} 
(MMAC) 2], 3]. This is because OFDM offers many advantages including high spectral efficiency and immunity to multipath fading and impulsive noise [1, 4].

However, OFDM-based systems are very sensitive to the frequency offset [5], which could destroy the orthogonal property among subcarriers, and consequently, deteriorate the overall performance of OFDM-based systems. Thus, the frequency offset estimation is one of the most important technical issues in OFDM-based systems [6], 7].

One of the main error sources in estimating the frequency offset in OFDMbased systems is the timing offset [4, 8]. Some techniques have been proposed to alleviate the effect of the timing offset on the frequency offset estimation [8]-[10]; however, most of the investigations require the knowledge of the timing offset range, which is not generally available in practical systems. Thus, it would be useful to develop an algorithm that does not require the knowledge of the timing offset range.

In this paper, we propose a new frequency offset estimation algorithm using differential combining between two successive correlation samples for OFDMbased WLAN systems. The timing offset causes a phase drift among the correlation samples used for frequency offset estimation, and thus, hinders the effective accumulation of the correlation samples, which is essential to obtain a large correlation value for the detection of the correct frequency offset estimate. In the proposed algorithm, we remove the phase drift due to the timing offset via differential combining between two successive correlation samples, and thus, can obtain a large correlation value for frequency offset estimation, regardless of the timing offset value. The simulation results show that the proposed algorithm is not only robust to the timing offset variation, but also performs better than the conventional algorithm on average, in the case of the timing offset range being not known exactly.

\section{Signal Model}

In OFDM-based systems, the transmitted symbol is generated by the inverse fast Fourier transform (IFFT) and expressed as

$$
z(m)=\sum_{k=0}^{N-1} Z_{k} e^{j 2 \pi \frac{k}{T_{s}} \frac{T_{s}}{N} m},
$$

where $m$ is the discrete time index, $Z_{k}$ is a phase shift keying (PSK) or quadrature amplitude modulation (QAM) data symbol of the $k$ th subcarrier, $T_{s}$ is the symbol period, and $N$ is the IFFT size.

For a zero-mean additive white Gaussian noise (AWGN) channel, the received signal can be expressed as

$$
y(m)=z(m-\tau) e^{j 2 \pi \Delta f(m-\tau) / N}+w(m),
$$

where $\Delta f$ is the frequency offset normalized to the subcarrier spacing $\frac{1}{T_{s}}, \tau$ is the timing offset normalized to the sample interval $T_{s} / N$, and $w(m)$ is the AWGN. For the sake of simplicity, $\Delta f$ is assumed to be an integer. 
The receiver first demodulates the received OFDM symbol using the FFT operation, and then, yields the FFT output, $Y_{l}$, corresponding to the $l$ th subcarrier as follows:

$$
Y_{l}=Z_{l-\Delta f} e^{\frac{-j 2 \pi(l-\Delta f) \tau}{N}}+W_{l},
$$

where $W_{l}$ is the FFT output of $w(m)$ corresponding to the $l$ th subcarrier. From (3), we can see that the FFT output of the received OFDM symbol is cyclically shifted by the frequency offset and its phase is rotated by the timing offset.

\section{The Effect of Timing Offset on Frequency Offset Estimation}

In this paper, we consider the frequency offset estimation system employing a training sequence, as in [8]-[10]. Then, an estimate $\Delta \hat{f}$ of $\Delta f$ can typically be obtained as

$$
\Delta \hat{f}=\arg \max _{d}\left\{\left|\sum_{k=0}^{N-1} Z_{k}^{*} Y_{(k+d)_{N}}\right|\right\},
$$

where $Z_{k}$ is the locally generated training sequence, $*$ denotes the complex conjugate, $d$ is the amount of cyclic shift, and $(\cdot)_{N}$ is the modulo- $N$ operator. When $\Delta f$ is estimated correctly, that is $\Delta \hat{f}=\Delta f$, the correlation value (normalized to $N\left|Z_{k}\right|^{2}$ ) in (44), if AWGN is not considered, becomes $\left|e^{\frac{-j \pi \tau(N-1)}{N}} \frac{\sin (\pi \tau)}{\sin (\pi \tau / N)}\right|$ and is plotted as a function of the timing offset $\tau$, as shown in Fig. 1. From Fig. 1, we can clearly observe that the correlation value used for the frequency offset estimation is very sensitive to the variation of the timing offset, which implies

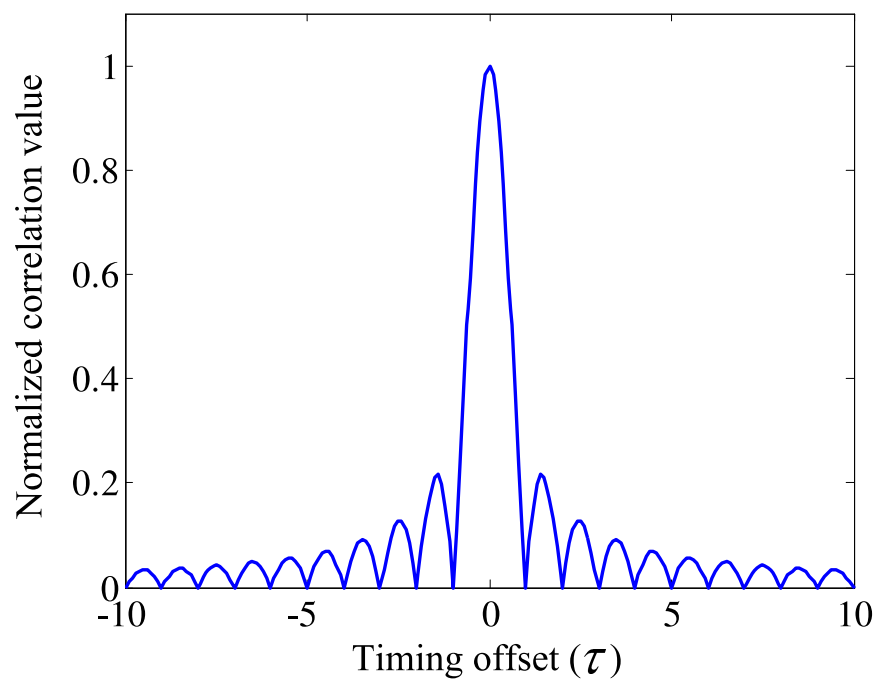

Fig. 1. Correlation value as a function of the timing offset $(N=1024)$ 


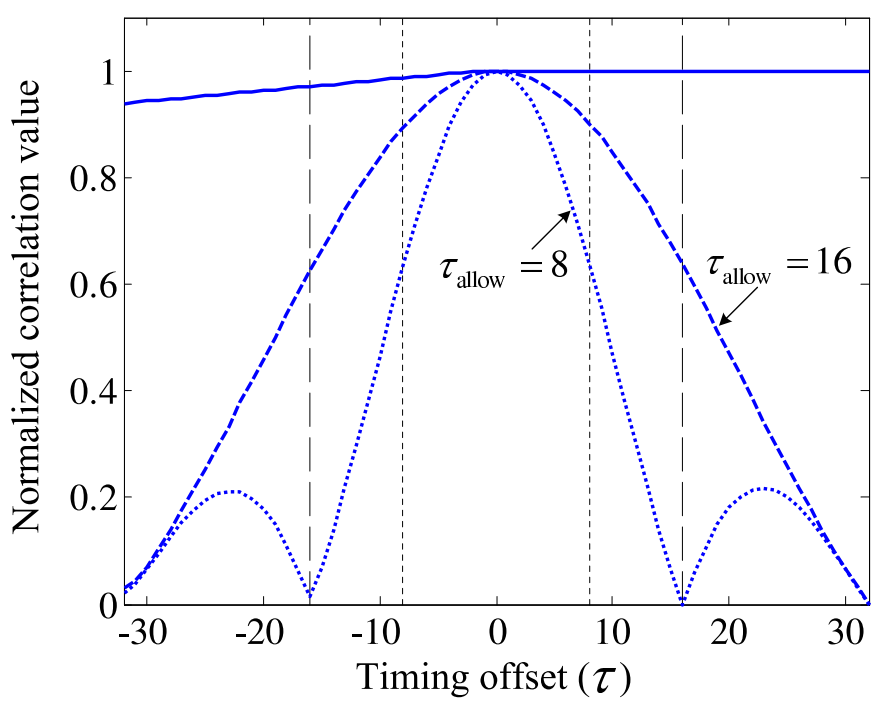

Fig. 2. Correlation value of the proposed (solid line) and conventional (dotted and dashed lines $)$ algorithms $(N=1024$, cyclic prefix $=100)$

that the correlation value could be reduced significantly due to the timing offset even if the frequency offset is correctly estimated, resulting in considerable degradation in estimation performance (note that a large correlation value when $\Delta \hat{f}=\Delta f$, is essential for the detection of the correct frequency offset estimate).

In the conventional algorithm, the frequency offset estimation in the the presence of the timing offset is achieved using the coherence phase bandwidth (CPB), which is the maximum correlation range in which the correlation value increases monotonically, and depends on the timing offset range strongly. Denoting the $\mathrm{CPB}$ and allowed timing offset by $B W_{c}$ and $\tau_{\text {allow }}$, respectively, we get

$$
B W_{c} \approx \frac{N}{2 \tau_{\text {allow }}}
$$

and using which we obtain the estimate $\Delta \hat{f}$ of $\Delta f$ as

$$
\Delta \hat{f}=\arg \max _{d}\left\{\sum_{n=0}^{K-1}\left|\sum_{k=0}^{B W_{c}-1} Z_{k+n B W_{c}}^{*} Y_{\left(k+n B W_{c}+d\right)_{N}}\right|\right\},
$$

where $K$ is the number of blocks divided by the $\mathrm{CPB}$ and $\tau_{\text {allow }}$ is equal to $N / B W_{c}$. As shown in (6), the conventional algorithm compensates for the correlation value reduction due to the timing offset by adding the absolute values of each partial correlation over $B W_{c}$. Fig. 2 shows the correlation value (normalized to $N\left|Z_{k}\right|^{2}$ ) in (6), where the dotted and dashed lines represent the cases of $\tau_{\text {allow }}=8$ and 16, respectively. As shown in the figure, the correlation value decreases rapidly as the timing offset becomes larger than $\tau_{\text {allow }}$, which results 
in the significant performance degradation in estimating $\Delta f$. That is, the conventional algorithm requires the knowledge of the timing offset range to set a value of $\tau_{\text {allow }}$ (or $B W_{c}$ ) demanded for its proper operation.

\section{Proposed Algorithm}

To mitigate the effect of the timing offset on the frequency offset estimation, we perform the differential combining between two successive correlation samples, $Z_{k}^{*} Y_{(k+d)_{N}}$ and $Z_{k+1}^{*} Y_{(k+1+d)_{N}}$. Then, the differentially combined components $\left\{\left(Z_{k}^{*} Y_{(k+d)_{N}}\right)\left(Z_{k+1}^{*} Y_{(k+1+d)_{N}}\right)^{*}\right\}_{k=1}^{N-1}$ become phase aligned, and thus, a large correlation value can be obtained by adding the components, regardless of the timing offset. It should be noted that each of the components $\left\{\left(Z_{k}^{*} Y_{(k+d)_{N}}\right)\right.$ $\left.\left(Z_{k+1}^{*} Y_{(k+1+d)_{N}}\right)^{*}\right\}_{k=1}^{N-1}$ is divided into real and imaginary parts. Hence, we take the envelope of $\sum_{k=0}^{N-1}\left(Z_{k}^{*} Y_{(k+d)_{N}}\right)\left(Z_{k+1}^{*} Y_{(k+1+d)_{N}}\right)^{*}$ to combine the divided parts, and finally, obtain the following frequency offset estimation algorithm:

$$
\Delta \hat{f}=\arg \max _{d}\left\{\left|\sum_{k=0}^{N-1}\left(Z_{k}^{*} Y_{(k+d)_{N}}\right)\left(Z_{k+1}^{*} Y_{(k+1+d)_{N}}\right)^{*}\right|\right\},
$$

From Fig. 2 in Section 3, we can see that the correlation value (normalized to $N\left|Z_{k}\right|^{2}\left|Z_{k+1}\right|^{2}$ ) of the proposed algorithm is almost constant regardless of the timing offset value. It is also observed that the correlation value of the proposed algorithm slightly decreases when the timing offset value is negative, which is caused by the interference from the neighboring preamble including the cyclic prefix, as shown in Fig. 3, where CP is an abbreviation of the cyclic prefix. However, from Fig. 2] we can see that the correlation value of the proposed algorithm is still much larger than that of the conventional algorithm.

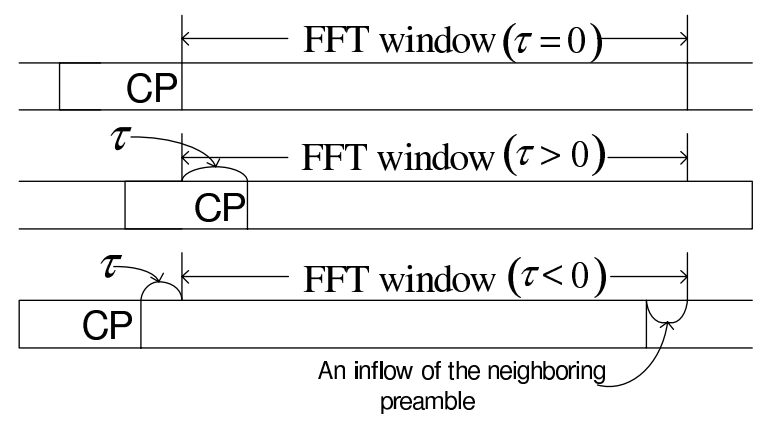

Fig. 3. Interference from the neighboring preamble when the timing offset value is negative 


\section{Performance Comparison}

In this section, we compare the performance of the proposed algorithm with that of the conventional algorithm [10 in terms of the frequency offset estimation accuracy, in the presence of the timing offset. Simulation was performed on an AWGN channel with a fixed frequency offset 10 and an OFDM system with a guard interval (cyclic prefix) of 100 samples and 1024 subcarriers was considered. The simulation results were obtained with $10^{3}$ Monte Carlo runs at each value of signal-to-noise ratio (SNR).

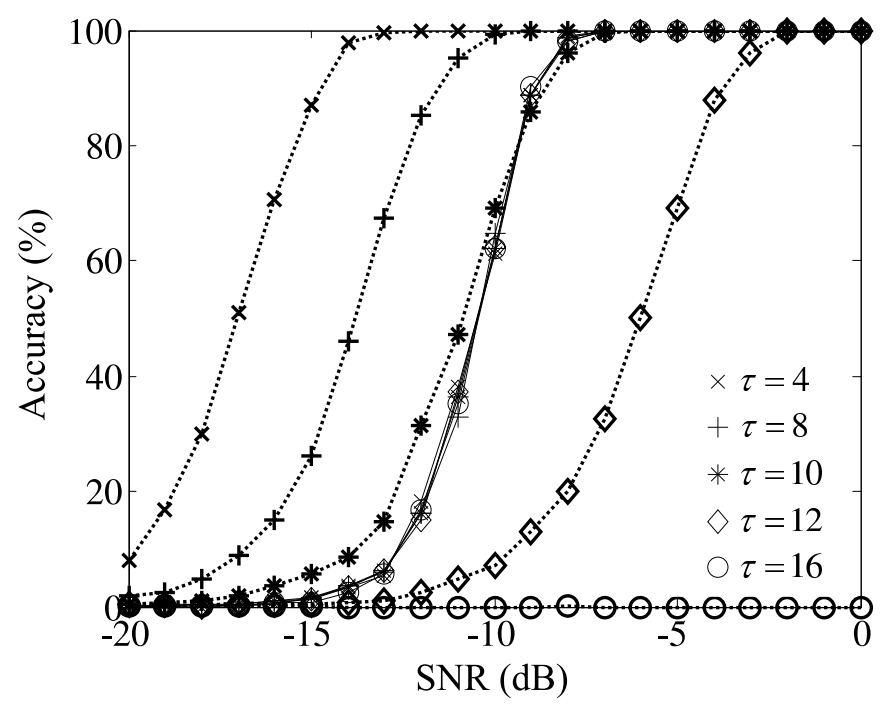

Fig. 4. Accuracy of the proposed (solid line) and conventional (dotted line) algorithms as a function of SNR when $\Delta f=10, N=1024, \tau_{\text {allow }}=8$, and $\tau=4,8,10,12$, and 16

Figs. 4 and 5 show the accuracy of the proposed (solid line) and conventional (dotted line) algorithms as a function of SNR when $\tau_{\text {allow }}=8$ and 16, respectively. From the figures, it is observed that the conventional algorithm performs better than the proposed algorithm when the timing offset $\tau$ is equal to or less than $\tau_{\text {allow }}$; however, the performance of the conventional algorithm significantly degrades as $\tau$ gets larger than $\tau_{\text {allow }}$ and eventually becomes much worse than that of the proposed algorithm. From the result, we can see that the conventional algorithm cannot operate properly without the knowledge of the timing offset range demanded for setting $\tau_{\text {allow }}$. On the other hand, it is seen that the proposed algorithm is much more robust to the timing offset variation and generally performs better than the conventional algorithm in practical systems, where the knowledge of the timing offset range is not available. 


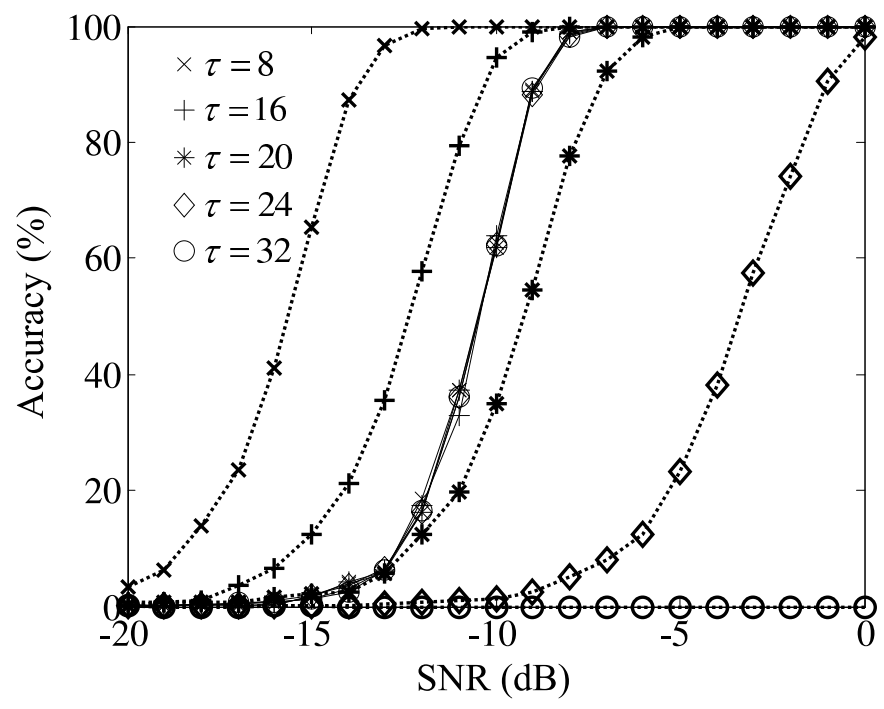

Fig. 5. Accuracy of the proposed (solid line) and conventional (dotted line) algorithms as a function of SNR when $\Delta f=10, N=1024, \tau_{\text {allow }}=16$, and $\tau=8,16,20,24$ and 32

\section{Conclusion}

In this paper, we first investigated the effect of the timing offset on the frequency offset estimation, and then, proposed to use the differential combining between two successive correlation samples to mitigate the effect. From the comparison results, we observed that the performance of the conventional algorithm might significantly degrade and eventually become useless for the frequency offset estimation in the case where the knowledge of the timing offset range is not available. On the other hand, the proposed algorithm is very robust to the timing offset variation and generally outperforms the conventional algorithm, in the case of the timing offset range being not known exactly.

\section{References}

1. R. V. Nee and R. Prasad, OFDM for Wireless Multimedia Communications. London, England: Artech House, 2000.

2. IEEE Std. 802.11a-1999, "Wireless LAN MAC and PHY Specifications - HighSpeed Physical Layer in the 5 GHz Band," ISO/IEC 8802-11: 1999 (E) / Amd 1: 2000 (E), New York, NY: IEEE, 2000.

3. N. Prasad and A. Prasad, WLAN Systems and Wireless IP for Next Generation Communications. Boston, MA: Artech House, 2002.

4. J. A. C. Bingham, "Multicarrier modulation for data transmission: an idea whose time has come," IEEE Commun. Mag., vol. 28, pp. 5-14, May 1990.

5. P. H. Moose, "A technique for orthogonal frequency division multiplexing frequency offset correction," IEEE Trans. Commun., vol. 42, pp. 2908-2914, Oct. 1994. 
6. B. Y. Prasetyo, F. Said, and A. H. Aghvami, "Fast burst synchronisation technique for OFDM-WLAN systems," IEE Proceedings: Commun., vol. 147, pp. 292-298, Oct. 2000.

7. J. Li, G. Liu, and G. B. Giannakis, "Carrier frequency offset estimation for OFDMbased WLANs," IEEE Signal Process. Lett., vol. 8, pp. 80-82, Mar. 2001.

8. H. Nogami and T. Nagashima, "A frequency and timing period acquisition technique for OFDM systems," in Proc. IEEE PIRMC, Toronto, Canada, pp. 10101015, Sep. 1995.

9. K. Bang, N. Cho, H. Jun, K. Kim, H. Park, and D. Hong, "A coarse frequency offset estimaion in an OFDM system using the concept of the coherence phase bandwidth," IEEE Trans. Commun., vol. 49, pp. 1320-1324, Aug.2001

10. S. Kim, S. Yoon, H. -K. Choi, and S. Y. Kim, "A low complexity and robust frequency offset estimation algorithm for OFDM-based WLAN systems," SpringerVerlag Lecture Notes in Compu. Sci., vol. 3992, pp. 961-968, May 2006. 